\title{
A Real-time Motion Tracking Wireless System for Upper Limb Exosuit Based on Inertial Measurement Units and Flex Sensors
}

\author{
S. S. Pastor, C. T. Rivera, O. F. Avilés*, M. F. Mauledoux \\ Mechatronics Engineering Program, Faculty of Engineering, Militar Nueva Granada University, Bogotá, Colombia
}

\section{$P A P E R \quad I N F O$}

Paper history:

Received 09 February 2018

Received in revised form 07 March 2019

Accepted 03 May 2019

\section{Keywords:}

Motion Tracking

Wearable Sensors

Upper Limb Exosuit

\begin{abstract}
A $B$ B S T R $R$ A $C$ C
This paper puts forward a real-time angular tracking (motion capture) system for a low cost upper limb exosuit based on sensor fusion; which is integrated by an elastic sleeve-mitten, two inertial measurement units (IMU), two flex sensors and a wireless communication system. The device can accurately detect the angular position of the shoulder (flexion-extension, abduction-adduction and internal-external rotation), elbow (flexion-extension and forearm supination-pronation), and wrist (flexion-extension) joints. In addition, the state of the hand (opened-closed). Finaly a PID controller is applied to the exosuit in order to replicate the movements performed by the arm into a 6 DOF robot arm.
\end{abstract}

doi: $10.5829 /$ ije.2019.32.06c.04

\section{INTRODUCTION}

Motion capture systems (MOCAP) are useful since they allow to obtain valuable information related to human locomotion, which may be analyzed for different purposes. For instance, to adopt strategies applied to rehabilitation processes, improve techniques to diagnose and treat neuromotor diseases, develop bio-inspired robots (humanoids) with better features and superior performance, enhance human computer interaction providing more intuitive and natural interfaces of control (e.g., operating a robot remotely with the movement of the user arm) [1,2], apart from other possible applications.

Exosuits of human limbs based on soft materials and the fusion of compact sensors (selected according to the type of joint, e.g., to measure elbow movement an adequate option is a bend/flex sensor, since elbow flexion only produces one degree of freedom. Otherwise, to track shoulder motion is useful to utilize an IMU because this joint has three degrees of freedom) that let augmenting mobility of the user without

* Corresponding Author Email: oscar.aviles@unimilitar.edu.co (O. F. Avilés) restricting natural kinematics or producing extra loads on it [3-6], are transforming the way in which the motion capture is made, bringing advantages over their predecessors with rigid body structures, such as low costs, light weight, improved comfort and simpler infrastructures $[5,7]$.

The present work is motivated by master-slave systems existing in robotics, which require intuitive interfaces of control to tele-operate slave machines, in this particular case, humanoid robots. The authors introduced a low cost intuitive mocap system for upper limb, Figure 1, that provides not only good performance, but also a proper transportable ergonomic structure that bring the possibility to control a manipulator remotely, in a simple and intuitive way.

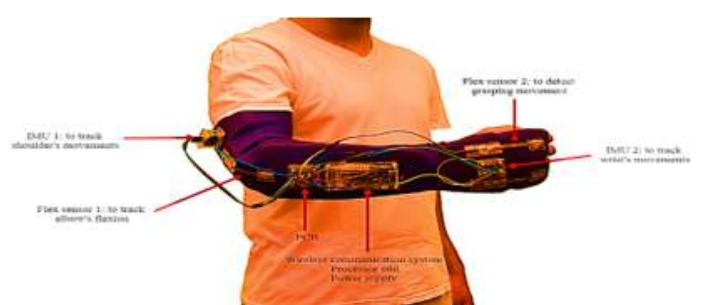

Figure 1. Prototype of proposed exosuit for motion tracking of human upper limb 
In general, a teleoperated system is the integration of a robotic device (slave) and a master device, which allows it to control the slave remotely. This class of systems are usually implemented when the task to be executed by the robot arm works autonomously presenting great complexity to itself or when the robot works in an environment that can implied a risk to the human being. In the case of robotic arms, this kind of platform are widely used to teach tasks by imitation of the human's motion or to execute tasks that required a highly precision but claims human's supervision as is the case of robots for surgery [8].

For these systems to be successful and to take advantage of them, it is required the development of an interface human-robot that allows an accurately control and a suitable data-feedback. An example of these interfaces thoroughly spread, with a large amount of acceptance in the research area are the haptic devices in virtual environments. Nevertheless, most of these devices are not intuitive enough to inexperienced users that do not have enough knowledge in the area of robotics [9]. On the other hand, among the most recent methods of teleoperation systems that provide a more natural integration to the user are those that allow direct mapping to the human's joints, in this case this method has been taken in care to the development of this work

\section{1. Architecture System Figure 2 shows a} functioning diagram of the teleoperation system, where it can be seen how the different elements interact based on the human being or user due to this one use the exoskeleton, which is in charge of measuring the motion of the different joints of human's upper limb. It sends the data collected to the computer application that manage the data (angular displacement related to each joint). In order to reply the human's motion in a 3D model of the robotic arm, this data is also sent to the microcontroller that have power over the manipulator.

\section{1. 1. Hardware Structure}

a) Upper-limb Exoskeleton: The implemented device, can measure the different degrees of freedom (DOF) based on the joints chosen of the human arm model stablished. This exoskeleton is lightweight, wireless and it has a good wearability being flexible.

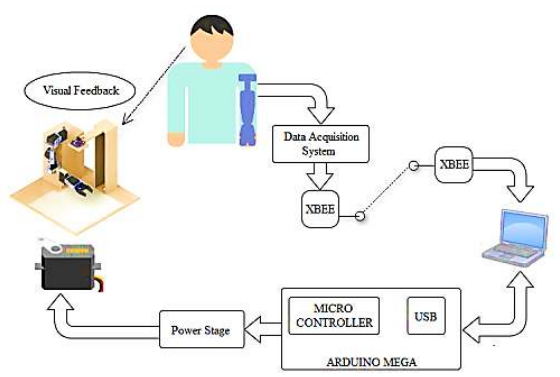

Figure 2. Functioning diagram of the teleoperation system b) Manipulator: The developed manipulator has 6 DOFs in order to be achieve any arbitrary positions and orientations due to the number of DOFs. This robot arm is called for general purpose. The distribution of the joints is similar to the human arm model stablished.

c) Human Arm Model implemented: The model applied has been based on Murray's kinematic model [9]; this one has 6 revolute DOF that are distribute as follows: three joints are located in the shoulder, one in the elbow and two in the wrist (the relation between this model and the human's arm is shown in Figure 3)

1. 1. 2. Software Framework The upper-limb exoskeleton sends to the computer application developed, the datum of the angular displacement of each joint, in order to replicate the movements performed in real time by the human arm on a $3 \mathrm{D}$ model placed into the app developed and a 6 DOF robot arm.

\section{MATERIALS AND METHODS}

The proposed exosuit is able to measure 6 DOF of human's upper-limb which are distributed as following: shoulder (flexion-extension, abduction-adduction and internal-external rotation), elbow (flexion-extension and forearm supination-pronation) and wrist (flexionextension) according to the kinematic model of Murray and the classification of arm motions made by Gopura.

The fundamental features taken into account to develop the device were portability, comfortability and adjustability, for this reason the structural material was required to be light and flexible. The design was based on the anthropometric measurements of the human body (Figure 4).

2. 1. Electronics

The electronic system is composed of a portable module and a PC module, as is

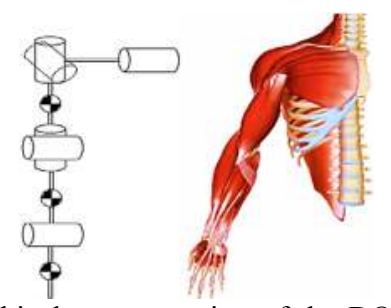

Figure 3. Graphical representation of the DOF related to the upper-limb
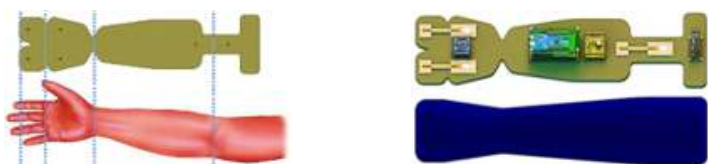

Figure 4. (a) Anatomy correlation between human arm and exosuit base structure in EVA foam, (b) Exosuit with all hardware attached and fabric sleeve-mitten to fit together user arm with exosuit 
presented in Figure 5. Portable module uses Arduino FIO (ATmega328V running at $8 \mathrm{MHz}$ ) as the processor to treat and calculate arm posture and joints displacement captured by bend and inertial sensors. PC module receives the information through serial port and reconstruct arm posture on a virtual application.

2. 2. Sensor location The proper location of the different sensors guarantees better measurements of the variables corresponding to angular displacements to the arm's joints. The device utilizes 2 IMUs of 9 DOF and 2 flex sensors of $50 \mathrm{~K} \Omega$. The first ones are used to measure the more complex joints (wrist and shoulder) and second ones sense 1 DOF (elbow flexion-extension and grasping movement).

IMU 1 is positioned in the back part of the arm and above the elbow (warranting non interference with its displacement) which allows to measure the mayor portion of shifting generated by the shoulder.

IMU 2 is placed on the dorsal of hand slightly below knuckles in order to sense the movements belonging to wrist, for this case, supination-pronation of forearm is associated to wrist since it has a superior impact on this joint, despite it is produced on the elbow.

Since flex sensors measure the amount of bending, they are located directly on the joints, elbow and metacarpophalangeal of index finger, respectively. In Figure 6, the distribution of sensors is portrayed.

2. 3. Validation Three different experiments were carried over proposed MOCAP system. In the first one,

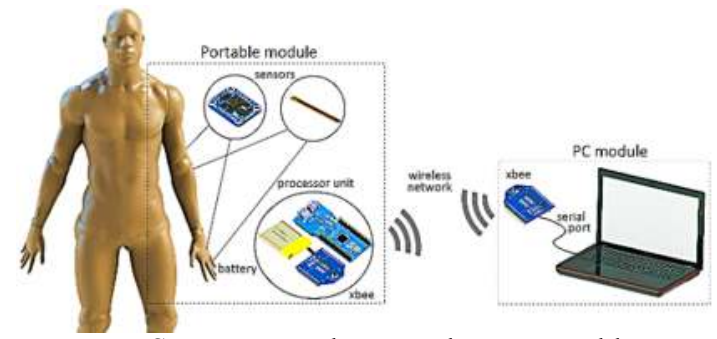

Figure 5. System topology, where portable module corresponds to hardware directly associated to exosuit and PC module is the destiny of captured data by exosuit (sent wirelessly) to be processed and displayed into a virtual application

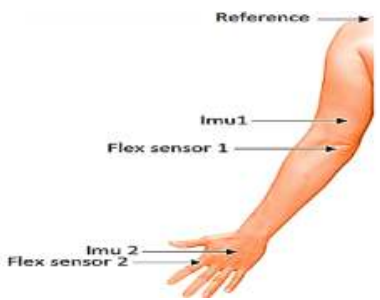

Figure 6. Sensors' location of exosuit in the arm frame. IMUs are positioned as farther of axis of rotation of joint (which is desired to track) as it is possible, whereas, flex sensors are placed straightly over the joint surface user wearing exosuit was asked to move its arm in all directions for 40 seconds, simultaneously, samples were recorded to apply the magnetometer calibration algorithm.

In the second, operator was asked to execute a pick and place task, inertial data coming from the most significant movement, in this particular case, wrist extension, was recorded to analyze fusion algorithm response (calculated angle) performance respect to individual angle estimation from gyroscope and accelerometer / magnetometer.

Finally, MOCAP system functionality is evaluated by comparing the estimated angles from soft exosuit against real angles measured from a digital goniometer. In order to achieve that user had to move its arm following a lineal trajectory, which comprises a total angular displacement of $90^{\circ}$.

\section{4. Calibration The obtained data from} experiment 1 is plotted as coordinates in Cartesian plane into axes couples $(x y, x z, y z)$ in Figure 7.

Angle measurement: Results of exosuit angle estimation performance are illustrated in Figure 8 (only two upper limb movements are plotted).

Fusion filter: Results of second experiment are plotted using Matlab as is shown in Figure 9.
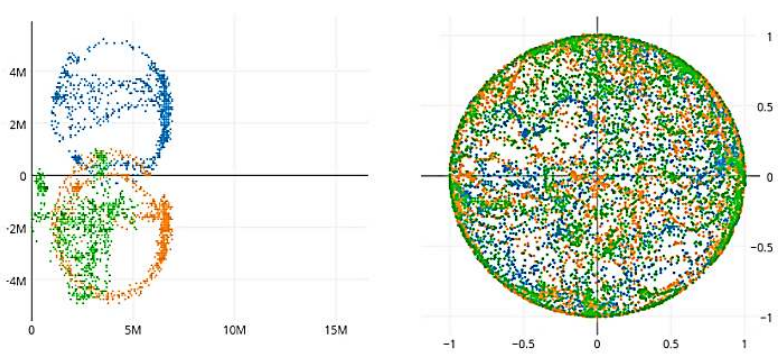

Figure 7. Projections on the plane of magnetometer tridimensional outputs plotted as coordinates. Previous calibration process (left picture), points of plane are scattered and remarkable away from the expected geometry, however, after calibration (right piture), the points of each plane describe a perfect circle centered in the origin

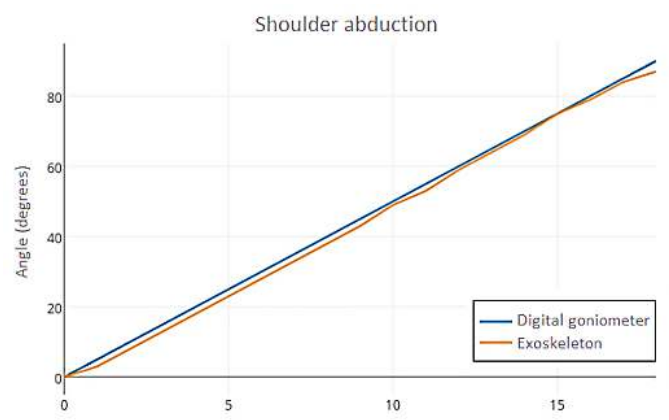

Figure 8. Exosuit and digital goniometer angular signal comparison in degrees. Blue line corresponds to goniometer output and orange line is the angle estimation from IMU 


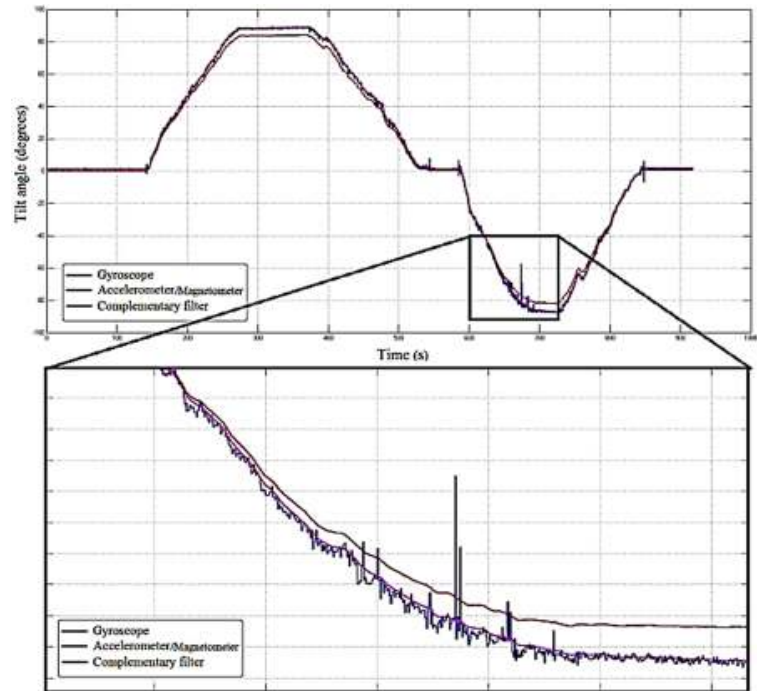

Figure 9. Complementary filter signal comparison. Red line is the gyroscope angle estimation, it describes a smooth signal that significantly diverges over the time and blue line corresponds to accelerometer/magnetometer angle estimation, whose signal has high frequency components as noise. Whereas, purple line corresponds to fusion algorithm response, which compensate the defects of previous estimations, generating an accurate angle calculation. All the values are in degrees.

\section{CONTROL DESIGN}

In order to control any system it is necessary to identify the dynamic of the plant (system), in simple terms, characterize the plant's response against a known input, which can be mathematical represented as a transfer function.

Since exist systems that are common on industrial or laboratory environments, some transfer functions' generic structures have been created with the purpose of synthetizing the general dynamic behavior of those mechanisms, this is the case of DC motors, whose dynamics is normally approximated to first or second order equations. Most of the time, first order expression is enough good to test control techniques.

The estimated transfer function that represents the studied actuators (DC motors) is shown in Equation (1),

$G p(s)=\frac{5,512}{s+9,87}$

The above expression relates an input of voltage to an output in terms of angular velocity. Nevertheless, the current work requires as output's variable the angular position, which can be easily obtained by integrating the response of the system or seen from other perspective by multiplying the transfer function by $\frac{1}{\mathrm{~s}}$ Equation (2).

$G p(s)=\frac{5,512}{s^{2}+9,87 s}$

Based on plant (DC motor) mathematical expression, controllers can be designed with the aim of forcing system's dynamics to performance according to desired parameters, settling time $\left(t_{s}\right)$ and signal overshoot percentage or $X_{i}(\xi)$, in the current case, values selected were $t_{s}=0,4 s$ and $\xi=0,95 \rightarrow M_{p}=1 \%$.

Different controllers following the general architecture presented in Figure 10 were tested to evaluate their properties to control the actuators (DC motors) of the virtual robotic arm in the most proper way (smoothly and robustly).

PID controller (continuous and discrete): Continuous PID is composed by analog electronic devices. Hence, it works on real time, but it is limited for the commercial values of the elements, for which in many cases is not possible to build the calculated circuits. On the other hand, discrete PID uses digital electronic, this is why there are not limitations in terms of parameters values, however a sample time is required in order to the controller be able to process the signals, which sometimes implicates getting powerful data acquirement systems.

These parameters can be fused in a single equation as portrays Equations (3) and (4) for analog and discrete time respectively, where $e(t)$ or $e(k)$ is the system error, $u(t)$ or $u(k)$ the control variable, $T_{d}$ the derivative time constant, $T_{i}$ the integral time constant

$$
\begin{aligned}
& u(t)=K_{p}\left[e(t)+T_{d} \frac{d}{d t} e(t)+\frac{1}{T_{i}} \int_{0}^{t} e(t) d t\right] \\
& u(k)=q_{0} e(k)+\frac{q_{1}}{1-z^{-1}} e(k)+q_{2} z^{-1} e(k)
\end{aligned}
$$

Plant cancellation controller: The aim of this controller is to cancel the dynamic of the plant in order to force the system to act the relation input-output is presented in Equation (5) from which is obtained the mathematical expression for the controller Equation (6), where $G_{p}(z)$ is the plant in time discrete, $D_{c}(z)$ is the controller, $R(z)$ is the input of the system and $C(z)$ is the output

$\frac{C(z)}{R(z)}=M(z)=\frac{D_{C}(z) G_{P}(z)}{1+D_{C}(z) G_{P}(z)}$

$D_{C}(z)=\left(\frac{1}{G_{P}(z)}\right)\left(\frac{M(z)}{1-M(z)}\right)$

In Equation (5), it can be noticed that the first factor cancels the plant and the second factor determine the response for the system. As a result of this process, it is required to choose a factor $M(z)$ that corresponds to the desired response.

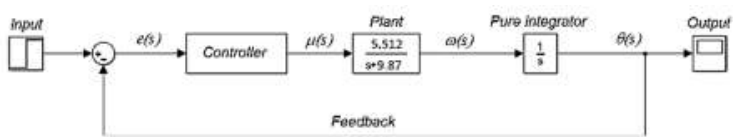

Figure 10. General control architecture for the DC motor system 
For this case the response of a first order system was chosen Equation (7), taking into account that $t_{s}=5 \tau$ and the sampling time $t_{m}=\frac{t_{s}}{40}$, equaling this expressions. We obtain a relation between $t_{m}$ and $\tau$ (Equation (8))

$M(z)=\frac{1-e^{-\frac{t_{m}}{\tau}}}{z-e^{-\frac{t_{m}}{\tau}}}$

$t_{m}=0.125 \tau$

Replacing Equation (7) into Equation (8), and this result into Equation (5).We obtain the transfer function in discrete time of the controller.

Control simulations: In order to validate the controllers' output over the DC motor application a model of them was implemented in Simulink of Matlab ${ }^{\circledR}$ as shown in Figures 11, 12 and 13.

The different control architectures were implemented on the manipulator's joints, the scheme shown in Figure 14. SimMechanics architecture implemented for the first joint, the other ones make use of the same structure, where it is necessary to declare for every link, the position of it, the center of mass and the type of joint (revolution or translation).

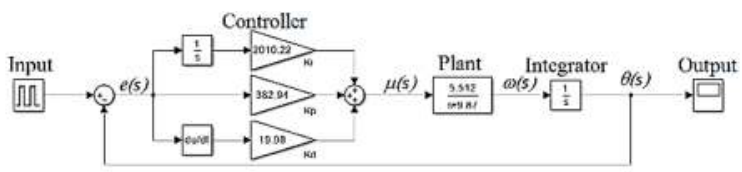

Figure 11. Simulink system model for the analog PID controller

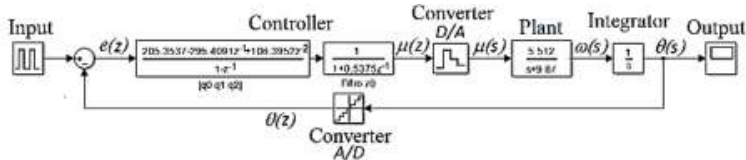

Figure 12. Simulink system model for the discrete PID controller

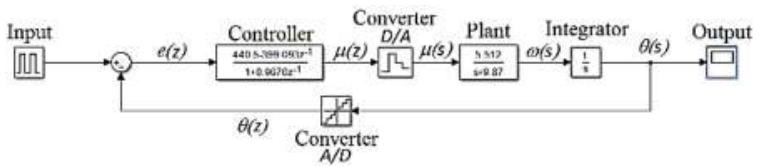

Figure 13. Simulink system model for the cancelling plant controller

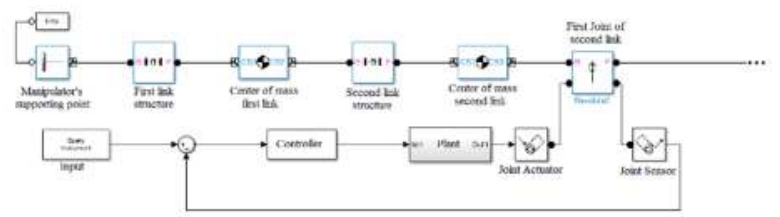

Figure 14. SimMechanics architecture for the first joint, it can be noticed where must be located the control system of each manipulator joint
The 3D model generated by SimMechanics of the manipulator is shown in Figure 15.

Results: The obtain data of the experiment is shown in Figures 16, 17 and 18, where are plotted the comparison between the input and the output of the system of each controller on one of the manipulator joints.

\section{EXPERIMENTS AND RESULTS}

The proposed system was validated (Figure 19) locating a goniometer in each joint and evaluating the concordance between the data received in the computer application and the value of the goniometer.

The deviation between these values were around $0^{\circ}$ to $3^{\circ}$ excluding the measure on the shoulder rotation whose value deviate around $0^{\circ}$ to $5^{\circ}$.

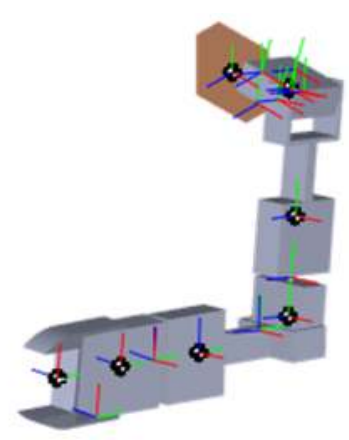

Figure 15. Simulated manipulator of 6 DOF on Simulink with SimMechanics



(a) System's input is a step signal

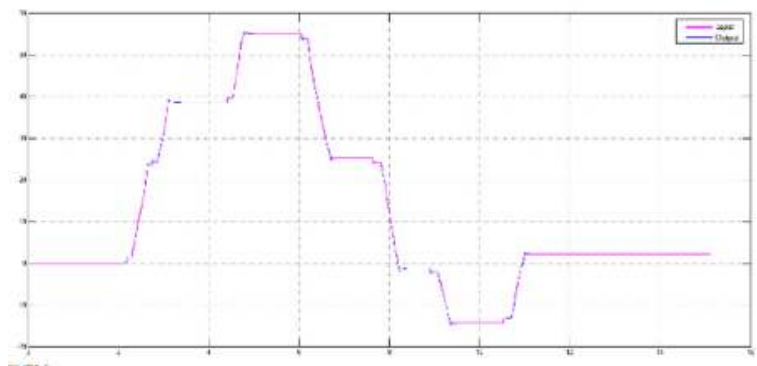

(b) Input is the data from exosuit

Figure 16. PID controller in continuous time performance evaluation 




(a) System's input is a step signal

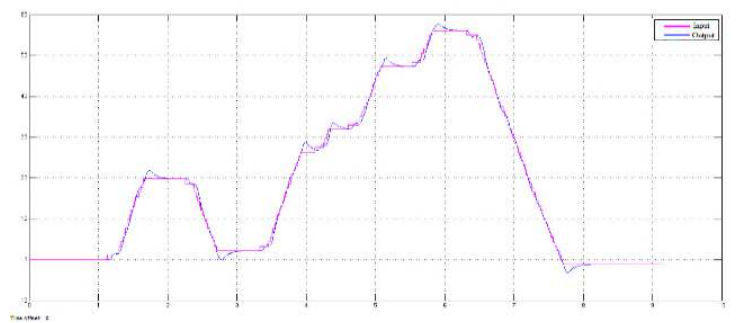

(b) Input is the data from exosuit

Figure 17. PID controller in discrete time performance evaluation time

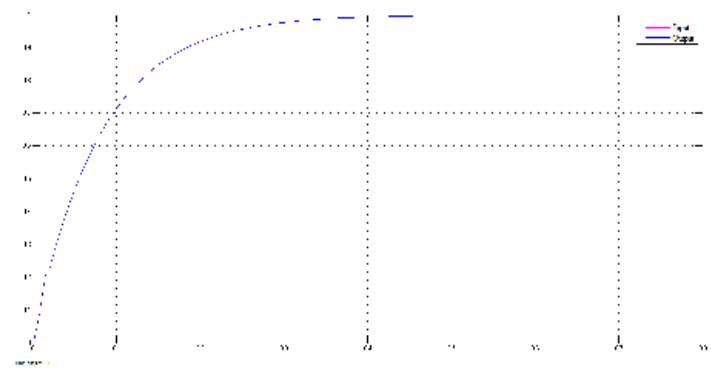

(a) System's input is a step signal

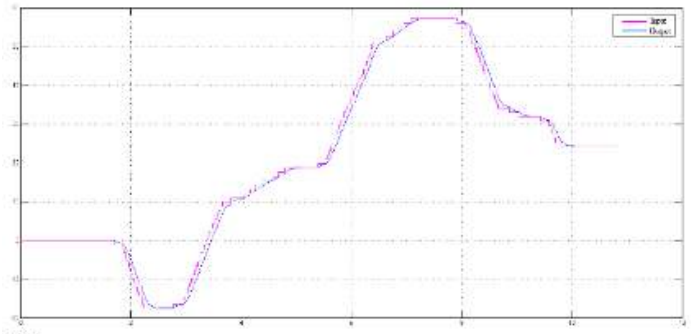

(b) Input is the data from exosuit

Figure 18. PID controller in continuous time performance evaluation

\section{DISCUSSION}

Magnetometer is probably the most sensible element belonging to inertial measurement unit, since it is affected by magnetic field of external devices (partially strong), in contrast to geomagnetic field (partially weak) which is detected by the sensor. For this reason, without a proper calibration, this sensor has a bigger tendency to present a large error, which can lead to issues in yaw angle estimation. The calibration algorithm used in this

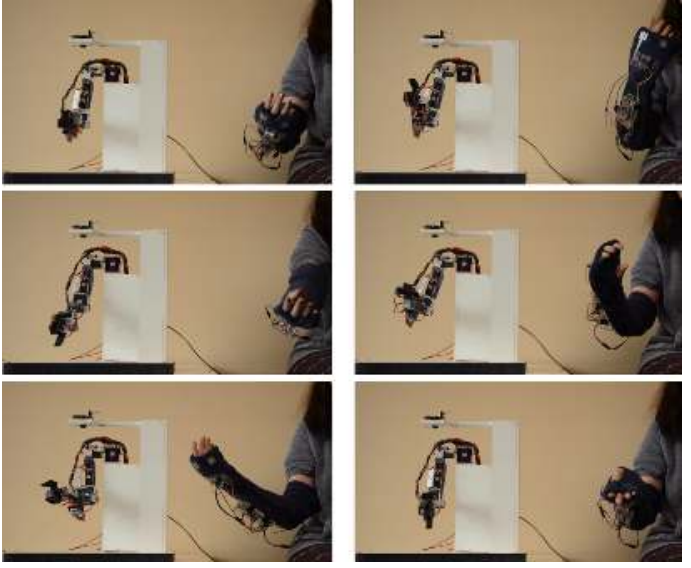

Figure 19. Movements validation.

work, not only considers the deviation of ellipsoid's center describes by magnetometer data, but also corrects the surface to transform it into an unitary sphere, this by implementing a briefer procedure against commonly used in literature $[10,11]$.

Although, first order complementary filter is not as accurate as other complex algorithms to calculate angular position based on inertial data from IMU, for instance, Mahony or adjustable Madgwick filter [12], with an average error below $1.1^{\circ}$, it is capable of estimating the angular displacement of the joints presenting an overall error of $3^{\circ}$ and a maximum error of $5^{\circ}$ in extreme positions, these results are similar to obtained data reported by Kalman-based algorithms [13$15]$ to inertial motion capture systems, whose average error is between $2^{\circ}$ and $5^{\circ}$ and are acceptable for many low cost applications.

The motion capture system developed in this work based on sensor fusion (IMU and flex sensors) to be used in exosuit for human's upper limb has an acceptable performance which presents enormous opportunities in different fields like tele-operation, medical or sport investigation, haptic interfaces, among others, due to its low cost, simple operation, transportability and easy implementation. Moreover, performance can be straightforward enhanced by replacing the fusion algorithm for one more robust as those mentioned before [5].

The implementation of plant cancellation controller, selecting as a desired response the output signal from a first order system, it gives promising results to control accurately angular position of DC motors, suppressing any possible overshoot on the signal. Additionally, settling time (delay) is enough short to permit develop a successful teleoperation process of the robotic arm on "real-time", following faithfully the reference signal from upper limb exosuit.

Cancellation plant technique offers some advantages over traditional PID controller, as avoiding experimental process like tunning to estimate a group of parameters that guarantees plant's stability, allowing 
gotten a desired response, regardless, plant's nature (order, grade, etc).

\section{CONCLUSIONS}

In this paper, a teleoperation system is developed. An upper-limb exoskeleton whose sensor system are IMU and flex sensor is implemented as a master device and a robot arm whose actuators are servomotors is the slave. The main components of the system related to hardware and software have been briefly introduced. The experimental result demonstrate that the master device can measure wearer's arm motion in order to correctly teleoperate the manipulator, due to the conversion implemented to change the measured motion to servomotor's range.

\section{ACKNOWLEDGEMENTS}

The present research paper was sponsored by Vicepresidency of Research at Nueva Granada Military University, through the project ING-2657 and Davinci research group

\section{REFERENCES}

1. Ju, Z. and Liu, H., "Human hand motion analysis with multisensory information", IEEE/AsMe Transactions on Mechatronics, Vol. 19, No. 2, (2013), 456-466.

2. Kobayashi, F., Kitabayashi, K., Nakamoto, H. and Kojima, F., "Hand/arm robot teleoperation by inertial motion capture", in 2013 Second International Conference on Robot, Vision and Signal Processing, IEEE., (2013), 234-237.

3. Mengüç, Y., Park, Y.-L., Pei, H., Vogt, D., Aubin, P.M., Winchell, E., Fluke, L., Stirling, L., Wood, R.J. and Walsh, C.J., "Wearable soft sensing suit for human gait measurement", The International Journal of Robotics Research, Vol. 33, No. 14, (2014), 1748-1764.

4. Seo, H. and Lee, S., "Design and experiments of an upper-limb exoskeleton robot", in 2017 14th International Conference on Ubiquitous Robots and Ambient Intelligence (URAI), IEEE. Vol., (2017), 807-808.
5. Brigante, C.M., Abbate, N., Basile, A., Faulisi, A.C. and Sessa, S., "Towards miniaturization of a mems-based wearable motion capture system", IEEE Transactions on Industrial Electronics, Vol. 58, No. 8, (2011), 3234-3241.

6. Marić, F., Jurin, I., Marković, I., Kalafatić, Z. and Petrović, I., "Robot arm teleoperation via rgbd sensor palm tracking", in 2016 39th International Convention on Information and Communication Technology, Electronics and Microelectronics (MIPRO), IEEE., (2016), 1093-1098.

7. Beigzadeh, B., Ilami, M. and Najafian, S., "Design and development of one degree of freedom upper limb exoskeleton", in 2015 3rd RSI International Conference on Robotics and Mechatronics (ICROM), IEEE. (2015), 223-228.

8. Austin, E. and Fong, C.P., "Teleoperated position control of a puma robot", (1987).

9. Grasshoff, J., Hansen, L., Kuhlemann, I. and Ehlers, K., "7dof hand and arm tracking for teleoperation of anthropomorphic robots", in Proceedings of ISR 2016: 47st International Symposium on Robotics, VDE., (2016), 1-8.

10. Mazinan, A.H., "High-performance robust three-axis finite-time attitude control approach incorporating quaternion based estimation scheme to overactuated spacecraft", International Journal of Engineering-Transactions A: Basics, Vol. 29, No. 1, (2016), 53-59.

11. McGinnis, R.S., Cain, S.M., Davidson, S.P., Vitali, R.V., McLean, S.G. and Perkins, N., "Validation of complementary filter based imu data fusion for tracking torso angle and rifle orientation", in ASME 2014 International Mechanical Engineering Congress and Exposition, American Society of Mechanical Engineers., (2014), V003T003A052V003T003A052.

12. Vasconcelos, J.F., Elkaim, G., Silvestre, C., Oliveira, P. and Cardeira, B., "Geometric approach to strapdown magnetometer calibration in sensor frame", IEEE Transactions on Aerospace and Electronic systems, Vol. 47, No. 2, (2011), 1293-1306.

13. Kok, M., Hol, J.D., Schön, T.B., Gustafsson, F. and Luinge, H., "Calibration of a magnetometer in combination with inertial sensors", in 2012 15th International Conference on Information Fusion, IEEE., (2012), 787-793.

14. Madgwick, S.O., Harrison, A.J. and Vaidyanathan, R., "Estimation of imu and marg orientation using a gradient descent algorithm", in 2011 IEEE international conference on rehabilitation robotics, IEEE., (2011), 1-7.

15. Öhberg, F., Lundström, R. and Grip, H., "Comparative analysis of different adaptive filters for tracking lower segments of a human body using inertial motion sensors", Measurement Science and Technology, Vol. 24, No. 8, (2013), 085703. 


\section{A Real-time Motion Tracking Wireless System for Upper Limb Exosuit TECHNICAL Based on Inertial Measurement Units and Flex Sensors

S. S. Pastor, C. T. Rivera, O. F. Avilés, M. F. Mauledoux

Mechatronics Engineering Program, Faculty of Engineering, Militar Nueva Granada University, Bogotá, Colombia

$P A P E R \quad I N F O$

جكيده

Paper history:

Received 09 February 2018

Received in revised form 07 March 2019

Accepted 03 May 2019

Keywords:

Motion Tracking

Wearable Sensors

Upper Limb Exosuit
اين مقاله يكى سيستم رديابى زاويه اى (سيستم ضبط حركتى) در زمان واقعى را براى يكى كم هزينه اكستروزن اندام فوقانى



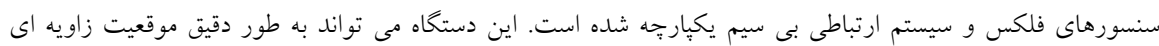



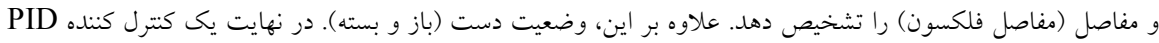

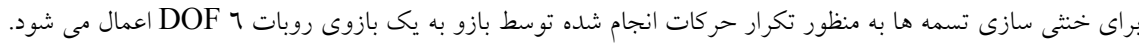
doi: 10.5829/ije. 2019.32.06c.04 